\title{
Qualidade de vida de pacientes em tratamento hemodialítico: intervenção da terapia ocupacional
}

\section{Quality of life of the patients in hemodialytic treatment: occupational therapy intervention}

\author{
Sônia Claudia Almeida Pinto', Lívia Mello Pontes² \\ http://dx.doi.org/10.11606/issn.2238-6149.v28i1p79-85
}

Pinto SCA, Pontes LM. Qualidade de vida de pacientes em tratamento hemodialítico: intervenção da Terapia Ocupacional. Rev Ter Ocup Univ São Paulo. 2017 jan.-abr.;28(1):79-85.

RESUMO: A doença renal crônica e a hemodiálise trazem ao paciente limitações, que afetam a qualidade de vida. O estudo objetivou descrever os resultados na qualidade de vida, após a intervenção terapêutica ocupacional com pacientes em hemodiálise em um setor de terapia renal substitutiva em Belém/PA e categorizar as atividades do programa de terapia ocupacional. O estudo é de tipo observacional, transversal, descritivo, analítico, tipo série de casos. Aplicou-se para avaliação da qualidade de vida o WHOQOL-Bref com 15 participantes e para guiar o plano terapêutico individual aplicou-se a lista de Identificação de Papéis Ocupacionais. O primeiro instrumento mensura a qualidade de vida em quatro domínios, o físico, o ambiental, o psicológico e o social. O segundo instrumento avalia o desempenho dos papéis ocupacionais ao longo do tempo. $\mathrm{O}$ WHOQOL-bref foi aplicado ao início e após as intervenções. Foi feita analise bioestatística por meio do software BioEstat versão 5.3. Como resultado observou-se aumento na auto avaliação da qualidade de vida de 7,2\% no domínio ambiental, de $12,8 \%$ no social, de $10,1 \%$ no psicológico e de 12,5 no físico, destacando melhoras significativas em todos os domínios, demonstrando a importância da abordagem do Terapeuta ocupacional junto ao paciente em tratamento hemodialítico.

DESCRITORES: Terapia ocupacional; Qualidade de vida; Hemodiálise.
Pinto SCA, Pontes LM. Quality of life of the patients in hemodialytic treatment: occupational therapy intervention. Rev Ter Ocup Univ São Paulo. 2017 Jan.-Apr.;28(1):79-85.

ABSTRACT: Chronic kidney disease and hemodialysis bring to the patient limitations, which affect the quality of life. The study aimed to describe the results on quality of life after occupational therapy intervention with patients on hemodialysis in a renal replacement therapy sector in Belém/PA and to categorize the activities of the occupational therapy program. The study is observational, transverse, descriptive, analytical, type of case series. The WHOQOL-Bref with 15 participants was used to evaluate the quality of life, and to guide the individual therapeutic plan, a list of Occupational Role Identification was applied. The first instrument measures the quality of life in four domains, the physical, the environmental, the psychological and the social. The second instrument assesses the performance of occupational roles over time. The WHOQOL-bref was applied at the beginning and after the interventions. A biostatistical analysis was carried out using the software BioEstat version 5.3. As a result, there was an increase in the self-rated quality of life of $7.2 \%$ in the environmental domain, $12.8 \%$ in the social area, $10.1 \%$ in the psychological and $12.5 \%$ in the physical, highlighting significant improvements in all The domains, demonstrating the importance of the Occupational Therapist approach to the patient undergoing hemodialysis.

KEYWORDS: Occupational therapy; Quality of life; Hemodialysis.

Monografia de conclusão de residência da terapeuta ocupacional Lívia Mello Pontes pela Universidade do Estado do Pará (UEPA/PA).

1. Doutora pelo Núcleo de Medicina Tropical da Universidade Federal do Pará (NMT / UFPA). Docente do Departamento de Terapia Ocupacional e pesquisadora do Núcleo de Pesquisa epidemiológica e Saúde Coletiva (NUPEC) do Centro de Ciências Biológicas e da Saúde da Universidade do Estado do Pará (CCBS/UEPA), Belém, Pará, Brasil. E-mail: salmeidapinto@bol.com.br

2. Especialista em atenção à saúde cardiovascular pela Residência multiprofissional no Hospital de Clínicas Gaspar Vianna (UEPA/ FHCGV). Terapeuta Ocupacional preceptora na Universidade da Amazônia. E-mail: liviapontes92@hotmail.com

Endereço para correspondência: Sônia Claudia Almeida Pinto. Gerencia de Ensino e Pesquisa do Hospital de Clinicas Gaspar Vianna. Tv. Alferes Costa, s/n - Pedreira, Belém - PA. CEP: 66087-660. 


\section{INTRODUÇÃO}

doença renal crônica é caracterizada pela
perda gradativa e irreversível da função
renal, que em seu último estágio é necessária a terapia renal substitutiva (TRS) que compreende a diálise peritoneal, hemodiálise ou transplante renal ${ }^{1}$. Dentre essas terapêuticas a mais utilizada no Brasil é a hemodiálise ${ }^{2}$.

No Brasil já se considera o número de pacientes em tratamento dialítico a nova epidemia do século XXI, o que torna a qualidade de vida e a sobrevida do paciente, cerne de estudos e elaboração de estratégias de atuação ${ }^{2}$.

$\mathrm{O}$ paciente que necessita de terapêutica hemodialítica, em geral, lida com um grande desafio que envolve mudanças em sua rotina e hábitos de vida, relacionados à alimentação, atividades físicas, medicações continuas e a dependência de pessoas e aparelhos ${ }^{3}$. Efeitos colaterais como náuseas e cefaléia também afetam a capacidade física necessária para o engajamento em seus papéis ocupacionais e melhoria da qualidade de vida ${ }^{4}$.

Neste sentido o Terapeuta Ocupacional (TO) pode contribuir com a melhoria da qualidade de vida, ao promover o aumento da funcionalidade, independência e autonomia nas atividades cotidianas, bem como buscar desenvolver mecanismos positivos para enfrentamento do adoecimento e do tratamento ${ }^{2,5}$.

No que concerne à intervenção junto aos pacientes em hemodiálise, o T.O procura estimular o engajamento, resgate ou ressignificação dos papéis ocupacionais que segundo Kielhofner são?

Papéis desempenhados todos os dias que influenciam a forma como a pessoa estrutura sua rotina diária. Estes não dependem apenas de habilidades para serem executados, mas são também influenciados por aspectos socioculturais, que definem quando, como, com quem e a frequência em que são realizados. As mudanças nos papéis ocupacionais afetam as atividades significativas na rotina. Este processo pode ocorrer no ciclo natural da vida, como mudança de estudante para trabalhador ou como consequência de condições clínicas como diagnósticos de doenças e seus tratamentos (p.35) .

Quando há alteração de papéis de forma abrupta ou sem a vontade própria do sujeito, podem ocorrer adoecimentos, baixa autoestima, perda da identidade e da rotina e prejuízo na qualidade de vida ${ }^{8}$.

Assim Madalosso e Mariotti ${ }^{3}$ sugerem como modelo teórico adequado para embasar as intervenções terapêuticas ocupacionais junto ao paciente em hemodiálise, o Modelo da Ocupação Humana (MOH). Que estuda a relação do sujeito e sua motivação para o envolvimento em ocupações. Este modelo mostrou-se útil na construção de um plano terapêutico singular voltado para a ocupação9. De acordo com o $\mathrm{MOH}$ os homens possuem uma natureza ocupacional. Sendo assim para que ocorra o envolvimento nas ocupações é essencial o equilíbrio de três subsistemas: vontade, habituação e capacidade de desempenho".

O subsistema vontade refere-se à motivação individual ou coletiva, estas incluem experiências de vida, sentimentos e objetivos de vida almejados. A habituação trata-se da capacidade de organizar suas ocupações em padrões e rotinas, este sofre grande influencia dos hábitos e papéis do individuo. E a capacidade de desempenho refere-se às habilidades físicas e mentais, ou seja, este necessita de todos os sistemas orgânicos para realizar suas atividades rotineiras?.

Nesse sentido foi elaborado por Francis Oakley Terapeuta Ocupacional em 1985, a Lista de Identificação de Papéis Ocupacionais como protocolo de avaliação para direcionar as intervenções de Terapia Ocupacional, fornecendo ao avaliador dados sobre a percepção do indivíduo em relação aos seus papéis ${ }^{10}$.

O instrumento é dividido em duas partes, onde a Parte I avalia, através de um tempo contínuo, os papeis de: estudante, trabalhador, voluntário, cuidador, serviço doméstico, religioso, amigo, passatempo/amador, membro de família e participante em organizações e outros ${ }^{10}$.

Na Parte II, identifica-se o grau de importância que o indivíduo atribui a cada papel relatado na Parte I, optando por uma das classificações a seguir: nenhuma importância, alguma importância ou muita importância ${ }^{10}$.

Um estudo realizado por terapeutas ocupacionais utilizando o protocolo supracitado em um setor de Terapia Renal Substitutiva evidenciou as contribuições da intervenção da Terapia Ocupacional embasada no $\mathrm{MOH}$ e nos papéis ocupacionais, apontou-se os benefícios referentes ao resgate papéis ocupacionais perdidos durante o tratamento ${ }^{7}$.

No que concerne à qualidade de vida e levando em consideração o tratamento hemodialítico optou-se pelo protocolo de avaliação de qualidade de vida WHOQOLBref (World Health Organization Quality of Life-bref) que possui características psicométricas. Este instrumento conta com 26 questões distribuídas entre 4 domínios: Físico, Psicológico, Relações sociais e Meio Ambiente ${ }^{11,13}$.

$\mathrm{O}$ estudo realizado na Fundação Hospital de Clínicas Gaspar Viana no Pará como parte da monografia de conclusão da Residência multiprofissional em saúde 
Pinto SCA, et al. Qualidade de vida de pacientes em tratamento hemodialítico. Rev Ter Ocup Univ São Paulo. 2017 jan./abr.;28(1):79-85.

cardiovascular trouxe como elemento motivador a prática em um hospital referencia em Nefrologia e Cardiologia.

Realizou-se revisão da literatura nas bases de dados LILACS, Pubmed, Medline e SciELO, aguçando ainda mais o interesse no estudo ao constatar a escassez de pesquisas que intercruzem a Terapia Ocupacional, hemodiálise e qualidade de vida. Sendo assim o presente estudo buscou compreender os impactos de um programa de Terapia Ocupacional na qualidade de vida de pacientes em tratamento hemodialítico.

A partir da ausência de literatura que descreva a atuação do terapeuta ocupacional na hemodiálise optou-se categorizar das atividades realizadas durante a hemodiálise para facilitar a compreensão por outros terapeutas ocupacionais das possibilidades de atuação em um ambiente com alto risco de contaminação e limitações físicas.

\section{PROCEDIMENTOS METODOLÓGICOS}

O estudo observacional, transversal, descritivo, analítico, tipo série de casos, com avaliação e reavaliação após período de intervenção, ocorreu no Setor de Terapia Renal Substitutiva (STRS) na Fundação Hospital de Clinicas Gaspar Vianna (FHCGV), hospital público de referência em nefrologia no estado do Pará, após aprovação no Comitê de ética de pesquisas com seres humanos da FHCGV com parecer de aprovação de número: 1.090. 686.

O tratamento de hemodiálise ambulatorial é dividido em turnos que seguem os seguintes horários: matutino $6 \mathrm{~h}$ às $10 \mathrm{~h}$; intermediário - $11 \mathrm{~h}$ às $15 \mathrm{~h}$ e vespertino/noturno - $16 \mathrm{~h}$ às 20h. Possuindo o intervalo de uma hora para a limpeza e desinfecção das máquinas de diálise.

\section{Universo e Amostragem}

Os sujeitos foram esclarecidos sobre as etapas da pesquisa e convidados a participar, a partir da assinatura do Termo de Consentimento Livre e Esclarecido (TCLE) a quem estivesse de acordo. Como critérios de inclusão foram selecionados sujeitos de ambos os sexos, portadores de doença renal crônica, em programa de hemodiálise ambulatorial, que não apresentassem déficit cognitivo nem problemas de comunicação verbal, de acordo com observações clínicas. Foram inclusos no estudo uma amostra de $n=15$ sujeitos que atendiam os critérios descritos.

\section{Instrumentos}

Para avaliação inicial e reavaliação da qualidade de vida utilizou-se o protocolo WHOQOL-Bref, versão abreviada do instrumento de avaliação de qualidade de vida da Organização Mundial da Saúde (OMS) o WHOQOL- $100^{13}$. Optou-se pela versão abreviada, por ser de fácil aplicação e compreensão. $O$ instrumento apresenta 4 qualificações para a qualidade de vida a partir da pontuação final da entrevista em cada domínio. Assim, a QV é considerada RUIM quando a pontuação for até 2,9, REGULAR, até 3,9, BOA até 4,9 e MUITO BOA, $5^{14}$.

Para guiar a elaboração do plano terapêutico individual e direcionar as intervenções adotou-se o instrumento Lista de Identificação de Papéis Ocupacionais versão em português. Trata-se de um protocolo validado de auto aplicação que avalia o desempenho de papéis ocupacionais em um tempo continuo e em relação a sua importância.

\section{Análise dos dados}

Para analisar a distribuição da qualidade de vida (WHOQOL-bref) e os papéis ocupacionais em uma amostra de $n=15$ pacientes foram aplicados métodos estatísticos descritivos e inferenciais. As variáveis quantitativas foram apresentadas por medidas de tendência central e foram comparadas pelo teste $\mathrm{t}$ de Student. As variáveis quantitativas apresentadas por distribuições de frequências absolutas e relativas e foram avaliadas pelo teste do Quiquadrado. A correspondência entre variáveis quantitativas foram apresentadas pela Correlação Linear de Pearson. Foi previamente fixado o nível alfa $=0.05$ para rejeição da hipótese nula. Todo o processamento estatístico foi realizado no software BioEstat versão 5.3.

\section{Procedimentos}

A coleta de dados ocorreu de setembro a novembro de 2015, iniciou com período de avaliação que perdurou um mês, utilizando o protocolo WHOQOL-bref para avaliação da qualidade de vida e da Lista de Papéis Ocupacionais para direcionamento dos planos terapêuticos individuais. Logo após, deu-se inicio ao período de intervenção que durou dois meses, após esse período reaplicou-se apenas o instrumento de avaliação da qualidade de vida. Todas as etapas foram empreendidas durante a sessão de hemodiálise.

No período de intervenção, cada um dos 15 sujeitos recebeu 10 atendimentos individuais, após cada sessão a mesma foi registrada em um diário de campo para posterior análise e categorização das atividades realizadas. As categorias elencadas foram construídas baseadas nos papéis ocupacionais observados na Lista de Identificação de Papéis Ocupacionais e foram agrupados por objetivos terapêuticos comuns. Como explanado na Tabela 1. 
Pinto SCA, et al. Qualidade de vida de pacientes em tratamento hemodialítico. Rev Ter Ocup Univ São Paulo. 2017 jan./abr.;28(1):79-85.

Tabela 1 - Categorização das atividades do programa de Terapia Ocupacional

\begin{tabular}{|c|c|c|}
\hline Categoria & Objetivos & Materiais /recursos \\
\hline Produtivo/Construtivo & $\begin{array}{l}\text { Direcionada aos indivíduos que apresentaram } \\
\text { interesse em resgatar seu papel ocupacional de } \\
\text { Trabalhador e Voluntário visa resgatar habilidades e } \\
\text { potencialidades. }\end{array}$ & $\begin{array}{l}\text { Revista, papel A4, tesoura sem ponta, cola, } \\
\text { barbante, feltro, linha, agulha, cartolina, retalho } \\
\text { de tecido, papel crepom, papel cartão, manta } \\
\text { acrílica. }\end{array}$ \\
\hline Lúdicas/Lazer/ Recreação & $\begin{array}{l}\text { Destinou-se aos sujeitos que referiram na lista } \\
\text { interesse em desempenhar o papel de Passatempo/ } \\
\text { Amador no STRS favorecendo atividades de lazer, } \\
\text { recreação em um ambiente onde antes permeava } \\
\text { apenas a ociosidade e a dor. }\end{array}$ & $\begin{array}{l}\text { Resta um jogo da memória, jogo da velha } \\
\text { adaptado com perguntas e respostas, Tangran, } \\
\text { quebra cabeças, damas, baralho, Lince, Mp3 e } \\
\text { fone de ouvido. }\end{array}$ \\
\hline Autoconfiança/ autoestima & $\begin{array}{l}\text { Manter a vontade de engajamento nos papéis, } \\
\text { esse tipo de demanda se apresenta na maioria dos } \\
\text { sujeitos da pesquisa. }\end{array}$ & $\begin{array}{l}\text { Maquiagem, materiais para limpeza de pele, } \\
\text { escova de cabelo, papel, lápis, revista, tesoura, } \\
\text { cola, Mp3 e fone de ouvido. }\end{array}$ \\
\hline Orientação em saúde & $\begin{array}{l}\text { Atividade avaliada como necessária a todos } \\
\text { os sujeitos como forma de compreensão e } \\
\text { esclarecimento sobre a doença, tratamento e } \\
\text { cotidiano. }\end{array}$ & $\begin{array}{l}\text { Papel, hidrocor, tesoura, cola, e cartilhas. } \\
\text { Em sua maioria foi utilizado apenas o recurso } \\
\text { humano através do diálogo. }\end{array}$ \\
\hline Práticas Corporais & $\begin{array}{l}\text { As atividades corporais na hemodiálise objetivam a } \\
\text { redução do estresse, e efeitos adversos comuns na } \\
\mathrm{HD} \text {, como a palpitação, dispneia e cefaleia. Bem } \\
\text { como facilita o processo de sono durante as sessões. }\end{array}$ & $\begin{array}{l}\text { Recurso humano para: Automassagem, } \\
\text { relaxamento direcionado, técnicas de respiração } \\
\text { profunda, técnicas de conservação de energia. }\end{array}$ \\
\hline
\end{tabular}

\section{RESULTADOS}

\section{Qualidade de vida de pacientes em tratamento hemodialítico}

O domínio Físico apresentou aumento de 12,5\% com p-valor $=0,0129^{*}$, conforme ilustrado no Gráfico 1 , onze dos quinze pacientes apresentaram aumento na auto avaliação da qualidade de vida no Domínio Físico, após as intervenções.

Gráfico 1 - WHOQOL-Bref domínio físico

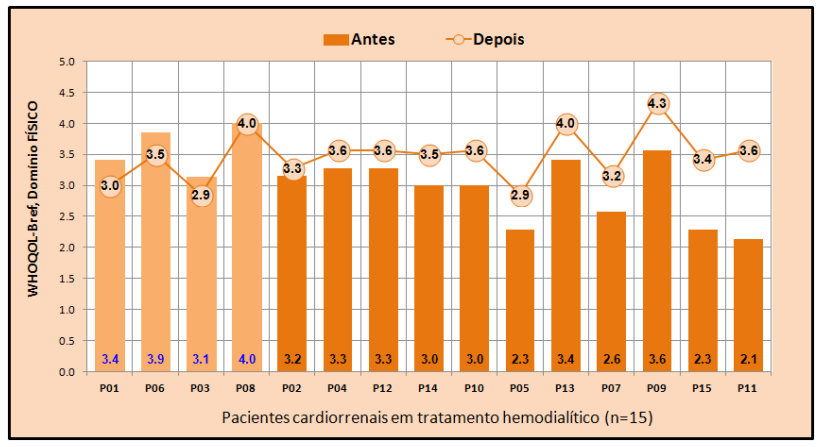

Fonte: Protocolo de pesquisa
Observou-se um aumento de $10,1 \%$ no domínio Psicológico após a intervenção com p-valor $<0,0001 *$, destaca-se que os pacientes P05, P11, P14 e P15 apresentavam antes da intervenção avaliação do domínio psicológico abaixo de 3, considerado Ruim e após a intervenção todos apresentaram aumento significativo, como observado no Gráfico 2.

Gráfico 2 - WHOQOL-Bref domínio psicológico

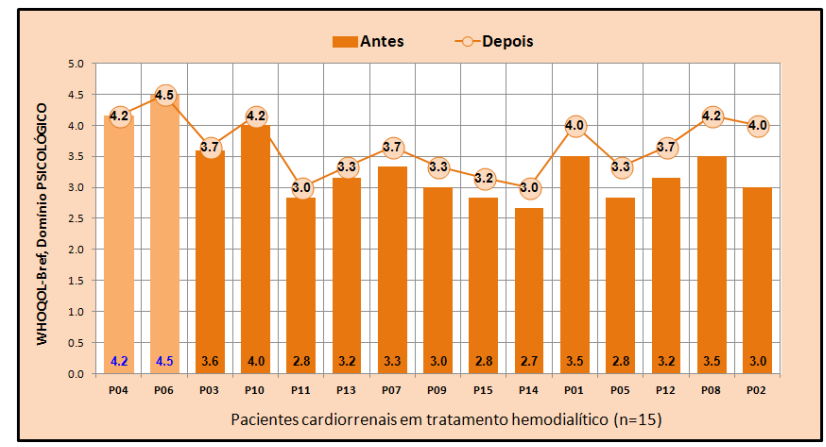

Fonte: Protocolo de pesquisa

No Gráfico 3 o domínio Social com p-valor $=0,0059^{*}$, apontou aumento de $12,8 \%$ após a intervenção. Onze dos quinze pacientes apresentaram 
aumento neste domínio, três mantiveram e apenas um reduziu nesta avaliação.

Gráfico 3 - Whoqol-Bref domínio social

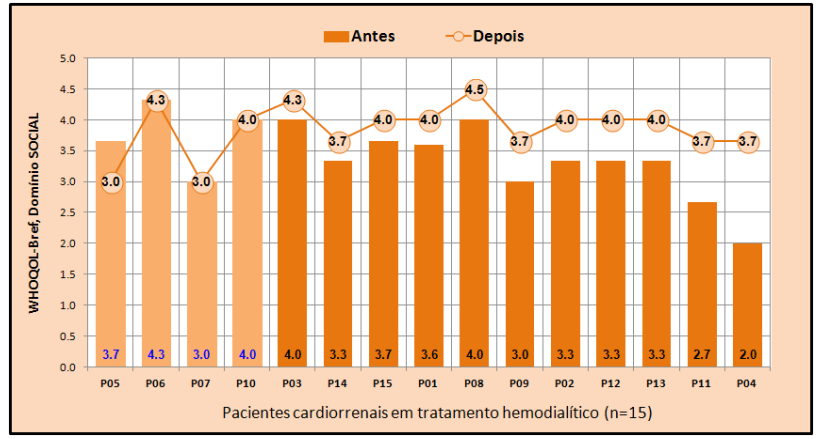

Fonte: Protocolo de pesquisa

No domínio Meio Ambiente o p-valor $<0,0001$ *, aumento de $7,2 \%$ após a intervenção. Vale ressaltar que neste domínio não houve redução da qualidade de vida, apresentando melhora em doze pacientes e mantendo em outros três (Gráfico 4).

Gráfico 4 - WHOQOL-Bref domínio ambiental

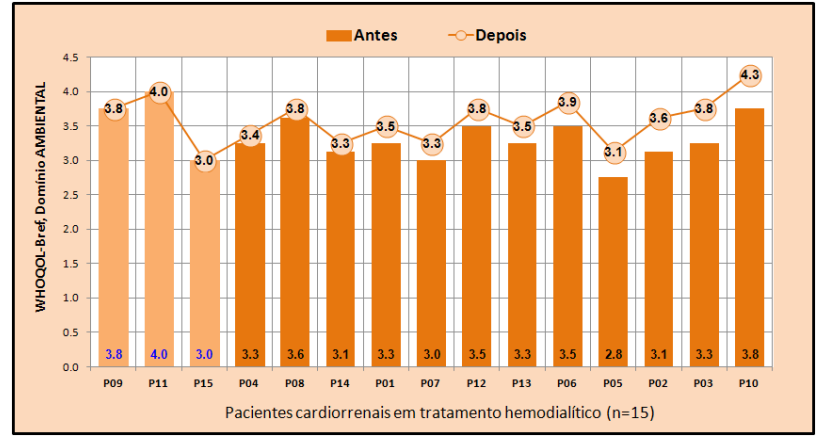

Fonte: Protocolo de Pesquisa

O Gráfico 5 demonstra o aumento estatisticamente significativo e finalizando com a qualidade de vida entre Regular e Boa em todos os Domínios avaliados pelo protocolo Whoqol-Bref, como descrito a seguir: domínio físico: $\mathrm{p}$-valor $=0,0129$ ( $\mathrm{t}$ de Student) aumento estatisticamente significante, domínio psicológico: p-valor $<0,0001$ (t de Student) aumento estatisticamente significante, domínio social: p-valor $=0,0059 \quad(\mathrm{t}$ de Student) aumento estatisticamente significante, domínio meio ambiente: $\mathrm{p}$-valor $<0,0001$ ( $\mathrm{t}$ de Student) aumento estatisticamente significante.
Gráfico 5 - Resultado da avaliação da qualidade de vida dos pacientes em TRS

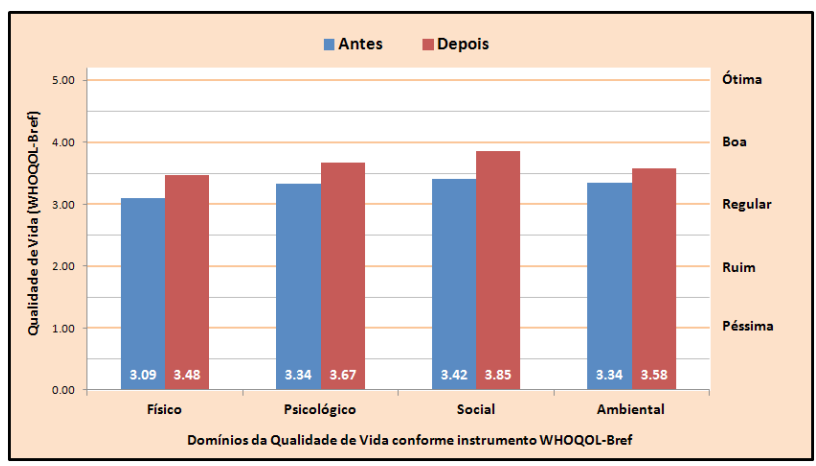

Fonte: Protocolo de pesquisa

\section{DISCUSSÃO}

Em relação à qualidade de vida no domínio físico antes das intervenções de Terapia Ocupacional, encontramos a qualidade de vida ente Ruim (até 2), Regular (até 3), Boa (até 4) e Ótima (até 5) dando destaque à $\mathrm{P} 05, \mathrm{P} 07, \mathrm{P} 11$ e P15 que avaliaram seu domínio físico com Ruim.

$\mathrm{O}$ aspecto físico é afetado diretamente pelo tratamento hemodiálitico, pelos efeitos colaterais mais comuns como "dor e fadiga" durante e após a terapêutica, assim como nos dias que antecedem a hemodiálise podem surgir edemas e fraquezas frequentes, o que acarreta em redução da capacidade física necessária ao desempenho de atividades cotidianas.

Resultados próximos ao do presente estudo, foram identificados na literatura onde o que apresentou índices menores de qualidade de vida foi o físico ${ }^{16}$.

Apesar da redução de energia e condicionamento físico observados em pacientes renais crônicos, pouco se encontra na literatura pesquisas e programas de orientação de exercícios para melhorar o condicionamento nas atividades cotidianas. Em uma pesquisa constatou-se que $33,3 \%$ dos pacientes afirmaram apresentar algum tipo de limitação física no cotidiano. Nesse contexto, os pacientes com IRC podem apresentar diminuição do desempenho funcional e prejuízo na prática de atividades físicas ${ }^{16}$.

Sobre as limitações físicas da presente pesquisa os sujeitos apresentaram arritmia, dor torácica e cefaleia, que são tratados com terapêutica medicamentosa pela equipe médica, porém aspectos comportamentais são observados decorrentes destes sintomas, como insônia e inquietação, estes aspectos foram abarcados nas atividades de Práticas 
Corporais e Orientação em Saúde favorecendo melhora dos efeitos comportamentais advindos dos sintomas físicos.

Essa melhora no desempenho físico pode ser observada na reavaliação, destacando individualmente que onze dos quinze pacientes obtiveram aumento da qualidade de vida neste domínio, os pacientes P15 e P11 tiveram os maiores aumentos saindo de QV Ruim para Boa, afirmando, portanto, efeitos positivos da intervenção.

Em relação ao domínio psicológico antes das intervenções mantiveram-se com conceitos, entre Ruim e Bom, de qualidade de vida, podendo dar ênfase aos pacientes P11, P15, P14 e P05 que apresentaram avaliação Ruim.

A literatura refere sobre os modos de enfrentar o tratamento hemodialítico, um positivo relacionado ao prolongamento da vida, e outro negativo relacionado à dependência de um tratamento para viver, infelizmente $o$ mais encontrado é o negativo, influenciando diretamente na vontade de realizar novas atividades, de reorganizar o cotidiano e de encontrar felicidade mesmo convivendo com uma doença ${ }^{14}$.

Os sentimentos de desanimo e desespero, podem estar associados as causas de abandono do tratamento, muitas vezes pela falta de orientação e suporte no inicio do tratamento. Esses sentimentos afetam o engajamento nos papéis de Estudante, Trabalhador e Passatempo, partindo do principio que todo envolvimento em qualquer atividade está sustentado na volição. Sem motivação, não existe nenhuma ação, já que o desejo de agir dá início à ação ${ }^{7}$.

Nesse sentido, as atividades produtivas, de lazer e orientações em saúde, propuseram também resgatar a volição baseadas na história individual. Os resultados podem ser observados na reavaliação às intervenções com aumento estatisticamente significante de 10,1\%, com destaque aos que haviam avaliado como Ruim o aspecto psicológico, na reavaliação os achados alcançaram conceito entre bom e ótimo.

O domínio social, no instrumento, avalia a rede de suporte do individuo, a capacidade de manter as relações sejam familiares ou de vínculos de amizade, apesar do adoecer. Pode-se associar nesse sentido o desempenho no papel de Amigo e Familiar. No WHOQOL os sujeitos apresentaram em sua maioria conceito entre Ruim e Bom. A partir disso a atuação direcionada à socialização e resgate de papéis de Amigo e Familiar, foram um dos focos das intervenções do presente estudo.

Resultado semelhante foi encontrado em um estudo sobre o estilo de vida de pacientes renais, eles possuíam, em sua maioria, boa relação com família, vizinhos e sociedade em geral. $\mathrm{O}$ autor acrescenta que rede de suporte social auxilia no enfrentamento da doença e de suas consequências, uma vez que ela integra o contexto no qual $\mathrm{o}$ indivíduo está inserido ${ }^{16}$.

Apesar de não desenvolver atividades com foco na socialização devido a boa avaliação do WHOQOLbref, o resultado de melhora de $12,8 \%$ na reavaliação, e a ausência de pacientes com conceito ruim, demonstrou como atividades terapêuticas ocupacionais no geral podem trazer melhoras de forma holística com esse público.

O domínio Meio Ambiente apresentou resultado satisfatório com apenas um paciente com conceito ruim na avaliação. Acerca da importância desse aspecto para a qualidade de vida, as condições ambientais e de moradia, barreiras físicas, facilidade de acesso à informação, levam o individuo à exercer sua cidadania e realizar suas atividades cotidianas, contribuindo para a qualidade de vida ${ }^{17}$.

Apesar de boa a avaliação inicial, a reavaliação deste domínio também apresentou aumentos na qualidade de vida de $7,2 \%$, refletindo efeitos positivos das intervenções voltadas ao aspecto ambiental. Após as intervenções nenhum paciente apresentou conceito ruim na qualidade de vida nesse domínio.

\section{CONCLUSÕES}

Foi possível ampliar as possibilidades de atuação do terapeuta ocupacional, bem como proporcionar ao paciente em hemodiálise um olhar mais holístico considerando suas demandas ocupacionais e de qualidade de vida, não centrado apenas no âmbito do medicamento. Quanto à literatura pouco foi encontrado de produção científica abordando a intervenção terapêutica ocupacional e seus efeitos na qualidade de vida de pacientes renais.

O estudo trouxe novas possibilidades de aprofundamento científico, identificando diversas demandas para futuros estudos no que tange a atuação e desenvolvimento de atividades dentro de um Setor de Terapia Renal Substitutiva e seus efeitos na qualidade de vida. 
Pinto SCA, et al. Qualidade de vida de pacientes em tratamento hemodialítico. Rev Ter Ocup Univ São Paulo. 2017 jan./abr.;28(1):79-85.

\section{REFERÊNCIAS}

1. Sette L, Titan S, Abensur H. Princípios básicos de nefrologia. São Paulo: Artmed; 2013.

2. Sesso R, Lopes A, Thomé F, Lugon J, Watanabe Y, Santos D. Diálise crônica no Brasil - relatório do censo brasileiro de diálise 2011. J Bras Nefrol. 2012;34(3):221-4. doi: 10.5935/0101-2800.20170001.

3. Madalosso F, Mariotti M. Terapia Ocupacional e qualidade de vida de pessoas com insuficiência renal crônica em hemodiálise. Cad Ter Ocup UFSCar. 2013;21(3):511-20. doi: http://dx.doi. org/10.4322\%2Fcto.2013.053.

4. Sesso R, Lopes A, Thomé A, Bevilacqua J, Romão Junior J, Lugon J. Relatório do censo brasileiro de diálise. J Bras Nefrol. 2008;30(4):233-8. doi: http://dx.doi.org/10.1590/ S0101-28002010000400007.

5. De Carlo M, Bartalotti C, Palm R. Terapia Ocupacional: reabilitação física e contextos hospitalares. São Paulo: Roca; 2004.

6. Terra F, Costa A. Nefrologia: uma abordagem multidisciplinar. Rio de Janeiro: Editora Rubio; 2011.

7. Santi A, Mariotti M, Cordeiro J. Lista de identificação de papéis. Rev Ter Ocup Univ São Paulo. 2012;23(3):289-96. doi: http://dx.doi.org/10.11606/issn.2238-6149.v23i3p289-296.

8. Brines E. Genesis da ocupação: um modelo filosófico para a terapia e a teoria. J Austr Ter Ocup. 1990;37(1):459. doi: http://dx.doi.org/10.11606/issn.2238-6149.v23i3p289-296.

9. Kielhofner G, Forsyth K, Kramer J, Melton J, Dobson E. Willard \& Spackman terapia ocupacional. Rio de Janeiro: Guanabara Koogan; 2011.

10. Colón H, Haertlein C. Spanish translation of the role checklist. Am J Occup Ther. 2002;56(5):586-9. doi: https:// www.ncbi.nlm.nih.gov/pubmed/12269515.
11. Fleck M. A avaliação da qualidade de vida: um guia para profissionais de saúde. Porto Alegre: Artmed; 2008.

12. The Whoqol Group. The World Health Organization quality of life assessment (WHOQOL): development and general psychometric properties. Soc Sci Med. 1998;12(1):1569-85. http://doi.org/10.1016/S0277-9536(98)00009-4.

13. Fleck M, et al. Desenvolvimento da versão em português do instrumento abreviado de avaliação da qualidade de vida “ WHOQOL- Bref”. Rev Saude Publica. 2000;34(2):178-83. doi: http://dx.doi.org/10.1590/S0034-89102000000200012.

14. Martins M, Cezarino C. Qualidade de vida de pessoas com doença renal crônica em tratamento hemodialítico. Rev Latino-am Enfermagem. 2005;13(5):670-6. doi: http:// dx.doi.org/10.1590/S0104-11692005000500010.

15. Rezende M. Tentando compreender o significado da hemodiálise para o paciente renal crônico a partir da fenomenologia existencial [Monografia]. São Paulo: Pontifícia Universidade Católica de São Paulo; 2006.

16. Kirchner R, Machado R, Löbler L, Stumm E. Análise do estilo de vida de renais crônicos em hemodiálise. Mundo Saúde (São Paulo). 2011;35(4):415-21. Disponível em: http://www.saocamilo-sp.br/pdf/mundo_saude/88/07 AnalisedoEstilodevida.pdf.

17. Ribeiro A, Sousa E, Atie S, Souza A, Shilitz E. A influência das quedas na qualidade de vida de idosos. Cienc Saude Coletiva. 2008;13(4):1265-73. doi: http://dx.doi. org/10.1590/S0104-11692005000500010.

18. Coelho D, Godoy C, Tavares H, Navarro F, Almeida E. Avaliação funcional e prescrição de treinamento para paciente portador de insuficiência renal crônica submetido a hemodiálise: um relato de caso. RBPFEx Rev Bras Prescrição Fisiol Exerc. 2007;1(3):29-41. doi: http://dx.doi. org/10.1590/S0104-11692005000500010. 